\title{
Can constant light exposure affect the thyroid gland in prepubertal male albino rats? Histological and ultrastructural study
}

\author{
F.A. Abdel Gawad, E.A.A. El-Shaarawy, S.F. Arsanyos, T.I. Abd El-Galil, G.N. Awes \\ Department of Anatomy and Embryology, Faculty of Medicine, Cairo University, Cairo, Egypt \\ [Received: 25 April 2018; Accepted: 24 June 2018]
}

Background: Through scientific literature, there is evidence that light affects thyroid function in human, mice and rabbits. Constant light and sleep deprivation is also used as a form of human torture, as it has impact on cognitive performances. The present work was conducted to study the effect of constant light for short and long periods on the thyroid gland in the prepubertal male albino rats.

Materials and methods: A total of 30 prepubertal male albino rats were used. The rats separated into three groups: group I (control); group II were those rats put under steady encompassing light (24 h/day, light intensity of 600 lux) for 4 weeks; and group III were the rats maintained in constant light for 3 months. The rat thyroid gland was subjected to histological and ultrastructural examination. Results: The rats exposed to light for long durations showed disturbed architecture; the follicles exhibited back to back arrangement (signs of hypertrophy with hyperplasia), lined by multiple layers of follicular cells or were lined by vacuolated cells. Few thyroid follicles exhibited cystic hyperplasia. Congested blood capillaries were demonstrated between the follicles.

Conclusions: It can be concluded that the short-term exposure to constant light for 1 month had no apparent effect on thyroid gland tissues while longer exposure to light for 3 months had detrimental effects on the thyroid gland structure of male albino rats. (Folia Morphol 2019; 78, 2: 297-306)

Key words: constant light, thyroid gland histological, prepubertal rats, thyroid gland ultrastructural

\section{INTRODUCTION}

The environmental 24-h light/dark cycle is involved in modulation of circadian rhythm in mammals. It regulates various biological activities including energy metabolism, body temperature, feeding, locomotion, and other types of behaviour $[4,8]$.

Hormone secretion is sensitive to time, which is necessary for achieving biological functioning. In mammals, the principal circadian mechanism is located in the suprachiasmatic nucleus of the hypo- thalamus. Through this nucleus directly or through pineal hormone melatonin coordinates the timing of circadian rhythms, including daily control of hormone secretion [21, 22]. Melatonin is one of the most studied biomarkers of the human physiological response to light [14]. The production of melatonin takes place almost exclusively at night. It has given rise to the concept that melatonin is the chemical expression of darkness. Continuous exposure to light leads to a complete suppression of melatonin release $[9,30]$.

Address for correspondence: Dr. E.A.A. Ahmed El-Shaarawy, Professor of Anatomy and Embryology, Department of Anatomy and Embryology, Faculty of Medicine, Cairo University, Kasr Al Aini Street, Cairo, Egypt, tel: 011201001582811 , e-mail: ehab.elshaarawy@yahoo.com; ehab.elshaarawy@kasralainy.edu.eg 
Melatonin supplementation inhibits thyroid growth, while pinealectomy stimulates it [3].

There is evidence in the scientific literature that light affects thyroid function in human [19], mice [2], and rabbits [40].

Vinogradova et al. [39] reported that exposure to constant light shortened average rat lifespan, increased incidence of metabolic syndrome development, accelerated spontaneous tumorigeneses and contribute to accelerated age [42]. Thyroid dysfunctions contributed to development of various pathological states. Studies demonstrated that thyroid hormones $(\mathrm{TH})$ and its receptors play a role in tumorigenesis and tumour progression $[7,16]$. In line with hypothesis of circadian disruption, light exposure at night disrupts the daily endogenous rhythm. Besides melatonin suppression, other mechanisms, including autonomic nervous system adjustment of different endocrine organ functions, may contribute to adaptation to constant light.

Constant light (CL) and sleep deprivation is also used as a form of human torture, as it has impact on cognitive performances [34].

Light at night became an essential part of modern lifestyle todays. Pollution with light, defined as excess artificial lighting during night-time can have detrimental effect on human health [29]. Thyroid gland is specialised for production, storage and release of thyroid hormones thyroxine (T4) and triiodothyronine (T3). T4 is the dominant hormone released from the thyroid gland, while $\mathrm{T} 3$ is biologically more active and originates mainly from peripheral deiodination of T4 [5]. Thyroid hormones play essential role in regulation of metabolic rate, regulation of growth, maturation of many organs and systems, including brain, skeleton, reproductive organs and energy expenditure [24, 37].

Rats prefer low light intensity and a well-controlled photoperiod will certainly contribute to stable circadian rhythms. Animal's activity and behaviour are also influenced by environmental intense light. Intense light conditions markedly suppress social play behaviour of Wistar rats, which is important for the animals' development [6]. Data on the effects of $C L$ exposure on pituitary thyroid axis are incomplete and mostly limited to determinations of serum hormone concentrations [39]. Little is known about the effect of light on the thyroid in humans. The normal thyroid-stimulating hormone (TSH) rhythm can be reset by a pulse of light [1]. The aim of the present work was to study the effect of constant light for short and long periods on the thyroid gland in the prepubertal male albino rats using histological and ultrastructural examinations.

\section{MATERIALS AND METHODS}

Animals

A total of 30 prepubertal male albino rats were used in this study. The body weight of the prepubertal rats (4 weeks old) ranged from 80 to $100 \mathrm{~g}$. Animal care was provided by laboratory animal house unit of Kasr Al-Ainy, Faculty of Medicine, Cairo University. The rats were acclimatised in the laboratory for a period of 2 weeks before carrying out the experiment. They were provided with ordinary rat chow and were housed in wire mesh cages (4 rats/cage) at a controlled temperature $\left(24 \pm 1^{\circ} \mathrm{C}\right)$, with normal light-dark cycle. The animals were given food and water ad libitum. The experiment was conducted in accordance with the guidelines of the committee of laboratory animals at Kasr El-Ainy School of Medicine.

\section{Experimental design}

All control rats were housed in standard stainless steel cages (4 rats per cage) with galvanised iron wires. The size of the control cages was $41 \times 28 \times$ $\times 19 \mathrm{~cm}$ allowing the animals to move freely. The custom-designed light experiment cage had a length, width, and height of $45 \mathrm{~cm}, 180 \mathrm{~cm}$, and $45 \mathrm{~cm}$; it was vertically divided into four equal chambers (length, width, and height of $45 \mathrm{~cm}, 45 \mathrm{~cm}$, and $45 \mathrm{~cm}$ for each chamber). A fluorescent lamp (light intensity: 600 lux) was installed in every chamber and had lights controlled by a switch that allowed free adjustment of the illumination time. The rats were divided into three groups.

\section{Group I (normal control)}

Ten prepubertal rats, 4 weeks old [27]. The rats were kept under regular laboratory lighting conditions (12-h light-12-h dark) during the same period. This was achieved by switching on the light at 7.00 in the morning and off at 7.00 in the evening. The rats were sacrificed according to the experimental schedule.

\section{Group II (exposed to constant light for short period)}

Ten prepubertal rats, 4 weeks old. The rats were placed into constant ambient light conditions (24 h/day, light intensity of 600 lux). The animals were maintained in constant light for 4 weeks [11]. 


\section{Group III (exposed to constant light for long period)}

Ten prepubertal rats, 4 weeks old. The rats were placed into constant ambient light conditions ( $24 \mathrm{~h} /$ day, light intensity of 600 lux). The animals were maintained in constant light for 3 months [27].

The rats of groups II (prepubertal, light exposure: up to 4 weeks) and III (light exposure: up to 12 week) nearly reaching the adulthood were exposed to light during the prepubertal period. The rats of group I were controls for group II and group III.

\section{Sacrification and thyroid extraction}

To reduce variance in the physiological parameters due to daily rhythms, all animals were sacrificed at the same time point in the circadian cycle, between 9:00 and 11:00 am. On the designated day, the rats were sacrificed by the end of 4 weeks for group II, 3 months for group III while the half of the control group were sacrificed after 4 weeks and the rest after 3 months. The animals were sacrificed under non stress conditions by rapid cervical decapitation. Their necks were opened through a ventral midline incision and the thyroid gland was rapidly dissected out and immediately weighed.

\section{Methods}

Thyroid specimens were obtained from all groups; one lobe was allocated for light microscopic examination and stained with haematoxylin and eosin (H\&E) and other stained with toluidine blue. The other lobe was allocated for ultrastructural examination using transmission electron microscope.

\section{RESULTS}

\section{Light microscopic results}

\section{Haematoxylin and eosin stained sections}

Group I. Histological examination of thyroid sections revealed thyroid follicles of different sizes; their cavities contained acidophilic colloid with peripheral vacuolations (Fig. 1a). Close observation revealed that the follicles were lined by cubical follicular cells that exhibited rounded nuclei. The follicles were separated from each other by narrow interfollicular spaces that contained minute blood capillaries (Fig. 1b).

Group II. The prepubertal rats exposed to constant light for short period demonstrated disturbed architecture of the thyroid lobule with large peripheral and small central thyroid follicles. Most of the follicles were filled with cracked colloid. Few follicles were filled completely with colloid and others showed peripheral vacuolations (Fig. 1c). Some fields showed dilated congested blood vessels (Fig. 1d-f). Some of the follicles exhibited several cellular layers while others were lined by flat cells (Fig. 1e).

Other fields confirmed widening inside the interfollicular spaces. A number of the follicles exhibited vacuolation of their cytoplasm (Fig. 1f).

Group III. The prepubertal rats exposed to constant light for long period demonstrated micro follicular thyroid follicles with small amount of colloid. The follicles were lined by tall columnar follicular cells with dark nuclei (Fig. 2a, b). Other fields showed disturbed architecture; the follicles exhibited back to back arrangement (signs of hypertrophy with hyperplasia), lined by multiple layers of follicular cells or were lined by vacuolated cells. Some follicles showed vacuolations of colloid (Fig. 2b, c). Few thyroid follicles were cystically dilated, some of them showed ingrowth projecting into the lumen and nearly obliterating it (Cystic hyperplasia) (Fig. 2c). Occasionally the thyroid follicles became adherent to each other with focal hyperplasia of the follicular cells that nearly obliterated their lumen (Fig. 2d, e). Congested blood capillaries were demonstrated between the follicles (Fig. 2a, b, c, e).

\section{Toluidine blue semithin stained sections}

Group I. Thyroid sections belonging to control prepubertal rats revealed thyroid follicles lined by one layer of cubical follicular cells with vesicular spherical nuclei, parafollicular cells with large pale nuclei and pale cytoplasm (Fig. 3a).

Group II. Thyroid sections belonging to group II rats revealed parts of thyroid follicles with increase in the height of cells lining the follicles. Most of these cells showed cytoplasmic vacuolations with either pale nuclei or pyknotic ones, whereas other follicular cells were ballooned with dark nuclei (Fig. 3b).

Group III. Thyroid sections belonging to group III revealed degenerated thyroid follicles containing desquamated epithelial cells in their lumen. Dark nuclei, vacuolated cytoplasm and dark flattened elongated nuclei of some follicular cells with colloidal vacuolations were noticed (Fig. 3c).

\section{Electron microscopic results}

Group I. Ultrastructural examination of the thyroid gland showed the follicular cells with euchromatic rounded or oval nucleus and prominent nucleolus. It was surrounded by regular nuclear membrane with nuclear pores. The cytoplasm contained organelles as mitochondria, rough endoplasmic reticulum, Golgi 


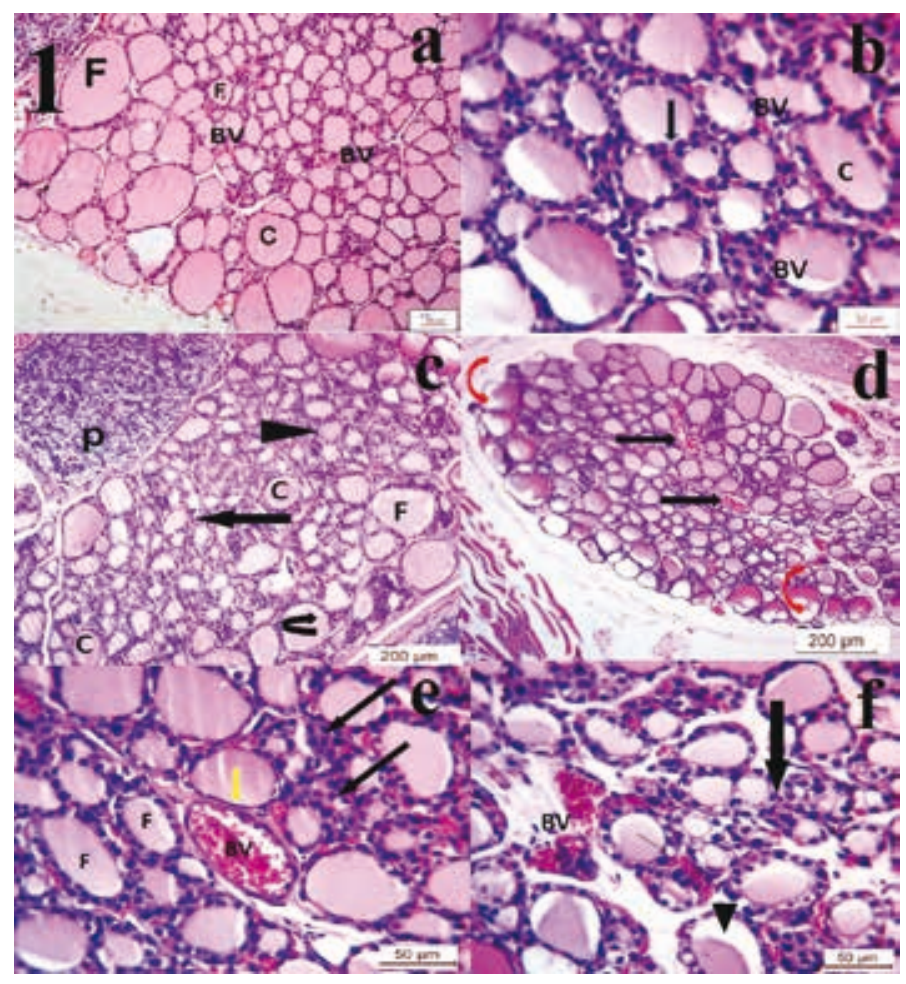

Figure 1. Micrograph of a section of the thyroid gland: a. Control prepubertal rat (group I) showing thyroid follicles (F) of variable sizes, filled with vacuolated acidophilic colloid $(C)$ and surrounded by interfollicular tissue containing blood vessels $(B V) ; \times 200 ; \mathbf{b}$. Control prepubertal rat (group I) showing thyroid follicles (F) lined by a single layer of cubical follicular cells exhibiting spherical vesicular nuclei (black arrow) and contain acidophilic colloid (C). The follicles are separated by narrow interfollicular spaces that contain blood vessels (BV); $\times 400$; c. Prepubertal rat (group II) showing disturbed architecture of the thyroid lobule with large peripheral (F) and small central thyroid follicles (black arrow). Most of the follicles are filled with cracked colloid (c). Few follicles are filled completely with colloid (arrow head) and others show peripheral vacuolations (curved arrow). Note the parathyroid gland $(p) ; \times 200 ; \mathbf{d}$. Prepubertal rat (group II) showing partial disruption of the thyroid lobule in the form of scalloping of the colloid in the thyroid follicles of the peripheral part of the thyroid lobule (red curved arrow). Note the dilated congested blood vessels in the centre of the thyroid lobule (black arrow); $\times 200 ;$ e. Prepubertal rat (group II) showing the thyroid follicles (F). Most of them are lined by a single layer of cubical cells. Some of the follicles exhibit several cellular layers (black arrow) while others are lined by flat cells (yellow arrow). Note a dilated congested blood vessel (BV) in the interfollicular space; $\times 400 ;$ f. Prepubertal rat (group II) showing widening in the interfollicular spaces. Most of the follicles are lined by single layer of cubical cells. Some of the follicles exhibit several cellular layers (black arrow) while others show vacuolation in the cytoplasm of the follicular cell (thin arrow). Some of the follicles show scalloping of colloid (arrow head). Note the ruptured blood vessels (BV). $\times 400$.

apparatus and electron dense granules. The follicular lumen showed fine granular colloid material with microvilli in the free surface of the follicular cells (Fig. 4a, b).

Group II. Ultrastructural examination of the thyroid sections belonging to group II rats revealed the follicular cells with undulation of nuclear membrane and margination of heterochromatin. The cytoplasm showed electron dense granules as compared to electron lucent granules, dilated rough endoplasmic reticulum and mitochondria with rupture of their outer membrane. The luminal surface showed multiple detached microvilli (Fig. 5a, b). Other fields showed heterochromatic nucleus with widely dispersed chromatin and prominent nucleolus. It was surrounded by irregular nuclear membrane. The mitochondria were swollen with disrupted cristae. Bundles of collagen fibres were demonstrated in the stroma (Fig. 5c). Some fields showed corrugated nucleus with clumping of heterochromatin. The follicular lumen contained fine granular colloid material with almost loss of microvilli on the luminal surface of cells (Fig. 5d). Other fields showed follicular cells with corrugated nucleus with thin rim of heterochromatin, dilated rough endoplasmic reticulum and colloid droplets in their cytoplasm. The luminal surface demonstrated elongated and detached microvilli (Fig. 5e).

Group III. Ultrastructural examination of the thyroid sections belonging to group III rats revealed 


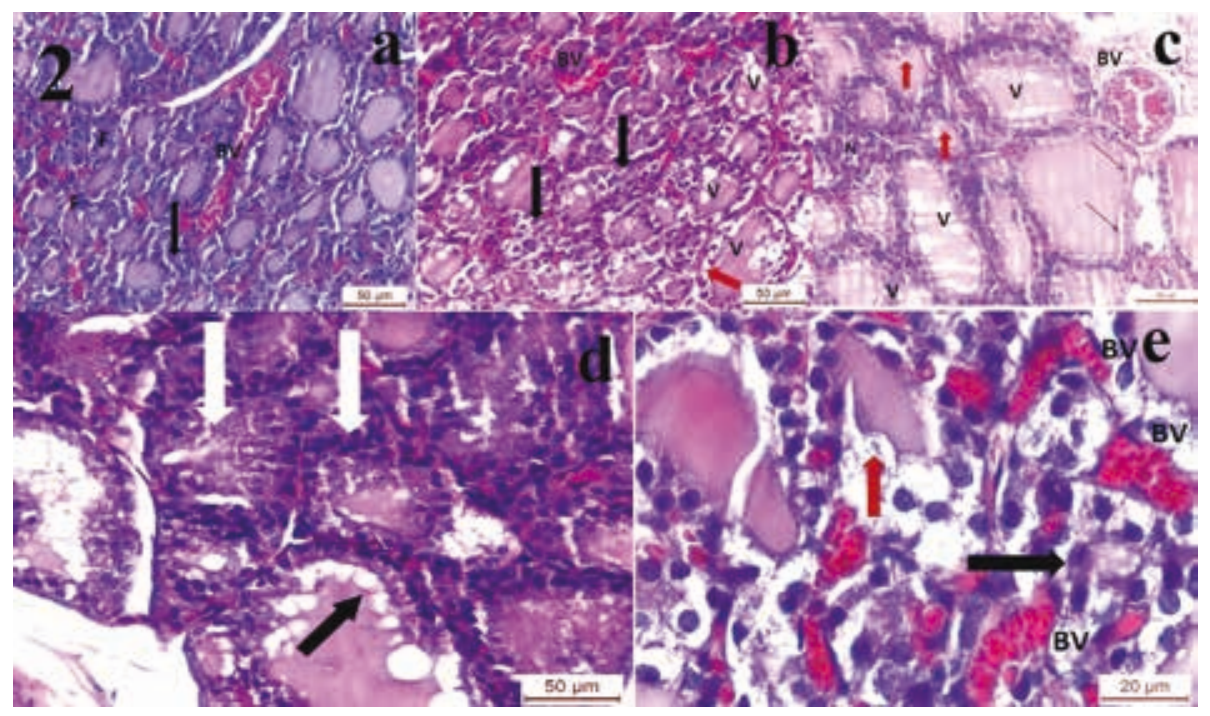

Figure 2. Micrograph of a section of the thyroid gland of a prepubertal rat of (group III) showing: a. Microfollicular thyroid follicles (F) with small amount of colloid. Note the congested blood capillaries between the follicles (BV). Some follicles show collapsed lumen (black arrow); b. Disturbed architecture of the gland; the follicles are lined by multiple layers of follicular cells in some parts (black arrow) and in other parts are lined by vacuolated cells (red arrow). Some follicles show vacuolations of colloid (v). Congested blood capillaries are seen (BV); c. Some thyroid follicles are cystically dilated, some of them show ingrowth projecting into the lumen (red arrow) and others exhibit multiple follicular cells with dark nuclei (N); they nearly obliterate its lumen. Note colloid resorption droplets (V), vacuolated cytoplasm (black arrow) and congested blood vessel (BV); d. Thyroid follicles become adherent to each other with focal hyperplasia of the follicular cells (white arrow). Note the vacuolated colloid (black arrow); e. Aggregate of small follicles lined by dark nuclei and having obliterated lumen (black arrow). Note congested blood vessels (BV) and retracted colloid (red arrow); $\times 400$.

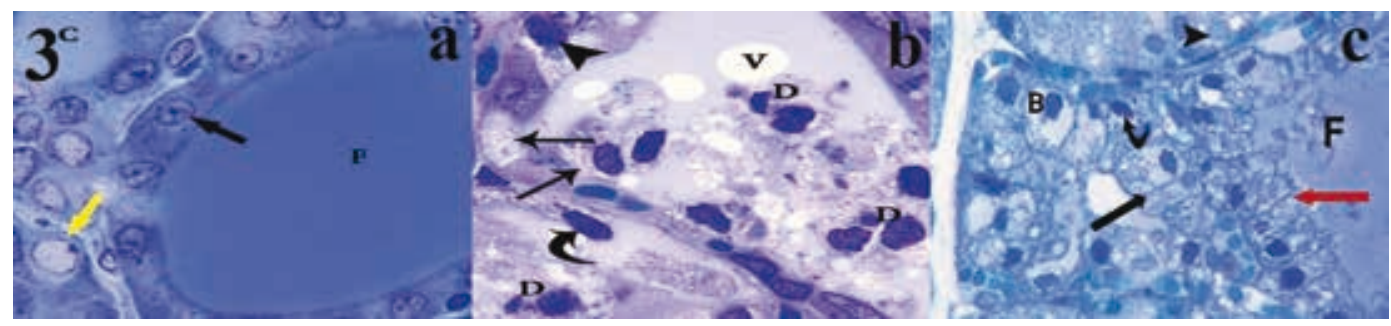

Figure 3. Micrograph of a semithin section in the thyroid gland of a prepubertal rat of (group I) demonstrating: a. Thyroid follicle (F) lined by one layer of cubical follicular cells with vesicular spherical nuclei (black arrows), parafollicular cells with large pale nuclei and pale cytoplasm (yellow arrow) are seen. Note the variable densities of colloid (C); $\mathbf{b}$. Degenerated thyroid follicles containing desquamated epithelial cells in their lumen (D). Dark nuclei (arrow head), vacuolated cytoplasm (thin arrows) and dark flattened elongated nuclei of some follicular cells are seen (curved arrow). Note the colloidal vacuolations (V); c. Parts of thyroid follicles (F). An apparent increase in the height of cells (red arrow) lining the follicles is seen. Most of these cells show cytoplasmic vacuolations (black arrow) with either pale nuclei (arrow head) or pyknotic ones (curved arrow), other follicular cells are ballooned with dark nuclei (B). $\times 1000$.

follicular cells with variably shaped hyperchromatic nuclei, swollen degenerated mitochondria and fragmented rough endoplasmic reticulum. Interfollicular capillaries containing RBCs in the stroma were detected (Fig. 6a). Some areas showed the follicular cells with rarefied cytoplasm (Fig. 6b). Electron-dense granules similar to lysosomes and fragmented dilated rough endoplasmic reticulum were demonstrated (Fig. 6c). Some fields showed detached apical microvilli in the lumen (Fig. 6a-c); others showed the follicular lumen with disrupted apical membrane (Fig. 6d). Other fields showed disrupted follicular cells with shrunken, condensed nuclei. Cytoplasmic vacuolations, fragmented rough endoplasmic reticulum and detached microvilli were exfoliated in the follicular lumen (Fig. 6e, f). Few fields showed exfoliation of some cells in the fine granular colloid material. The exfoliated cells showed condensation of the nuclear chromatin with distorted, variable shapes of nuclei and marked reduction of cell organelles (Fig. $6 \mathrm{~g}$ ). Thin bundles of collagen fibres were demonstrated in the stroma (Fig. 6f, g). 


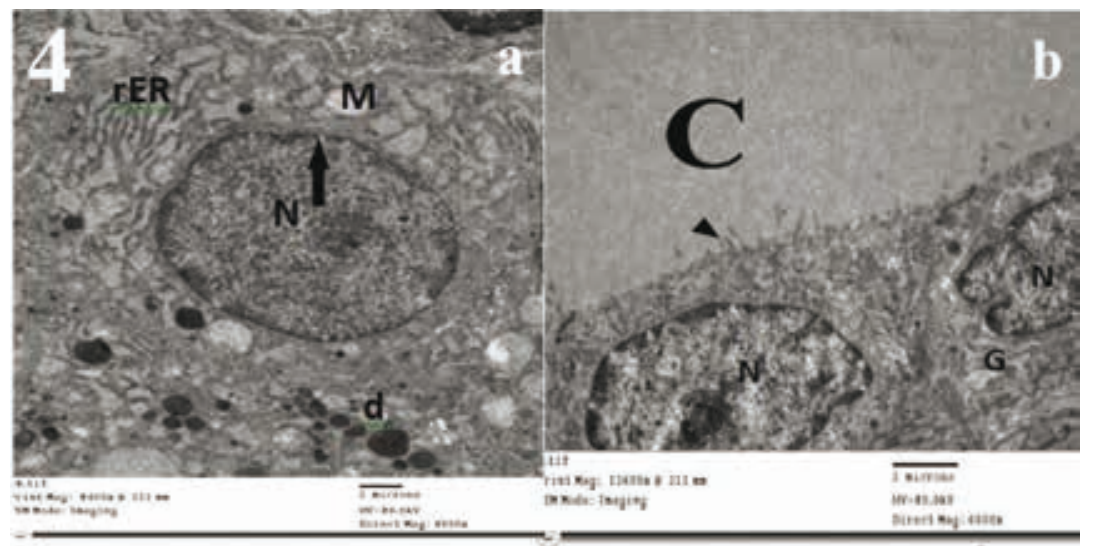

Figure 4. An electron micrograph of the thyroid gland of control prepubertal rat group I showing: a. A follicular cell with euchromatic nucleus (N) and prominent nucleolus. It is surrounded by regular nuclear membrane with nuclear pores (black arrow). The cytoplasm contains organelles as mitochondria $(M)$, rough endoplasmic reticulum $(r E R)$ and electron-dense granules $(d) ; \times 4000 ; \mathbf{b}$. Follicular cells with euchromatic nucleus (N) and prominent nucleolus. The follicular lumen shows fine granular colloid (C) material. Note Golgi apparatus (G) and the microvilli (arrow head) in the free surface; $\times 6000$.

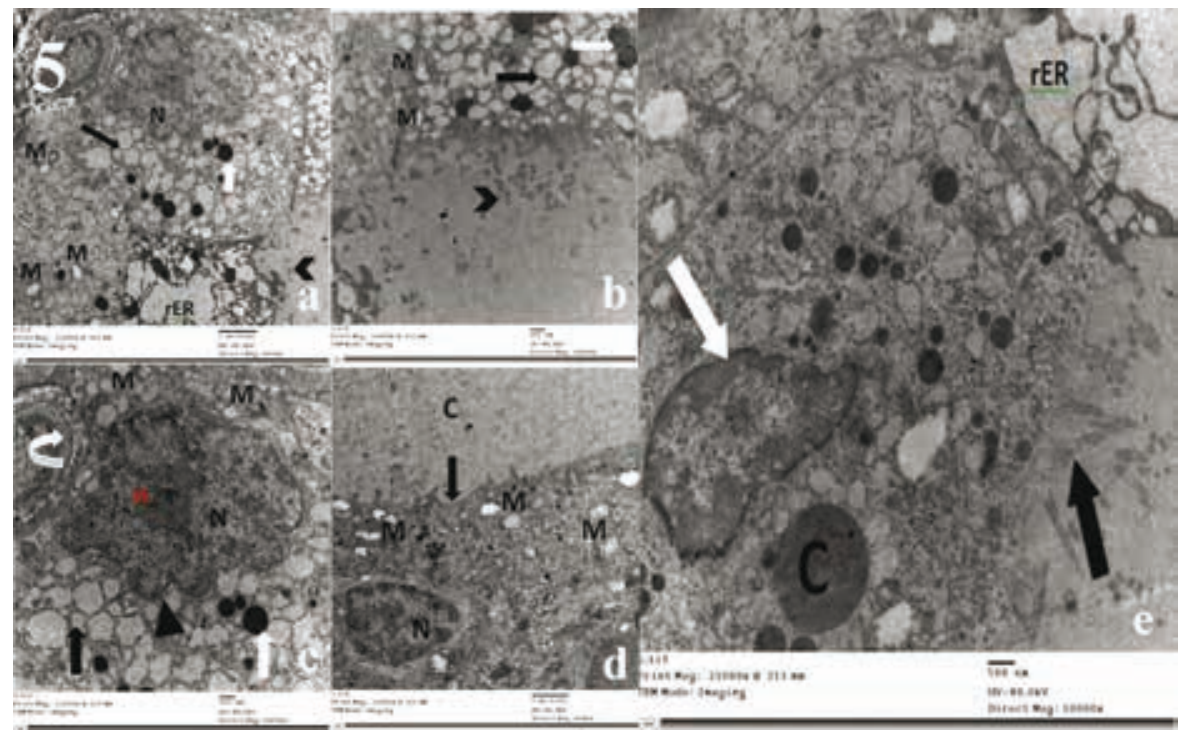

Figure 5. An electron micrograph of the thyroid gland of a prepubertal rat of group II showing: a. Electron dense granules (white arrow) as compared to electron lucent granules (black arrow). The luminal surface shows multiple detached microvilli (arrow heads). The mitochondria (M) are relatively swollen with rupture of their outer membrane. Note the dilated rough endoplasmic reticulum (rER), undulation of nuclear membrane and margination of heterochromatin $(\mathrm{N}) ; \times 6000 ; \mathbf{b}$. Electron dense granules (white arrow) as compared to electron lucent granules (black arrow) and swollen mitochondria with ruptured cristae are seen (M). The luminal surface demonstrates numerous detached microvilli in the lumen (arrow head); $\times 10,000$; c. Heterochromatic nucleus (N) with widely dispersed chromatin and prominent nucleolus (n). It is surrounded by irregular nuclear membrane (arrow head). The mitochondria are swollen with disrupted cristae (M), electron lucent (black arrow) and electron dense (white arrow) secretory granules are shown. Note bundle of collagen fibres (curved arrow); $\times 10,000 ; \mathbf{d}$. Corrugated nucleus (N) with clumping and margination of heterochromatin. The mitochondria are swollen with disrupted cristae. The follicular lumen contains fine granular colloid material (C) with almost loss of microvilli on the luminal surface of cells (black arrow); $\times 6000 ;$ e. A follicular cell with colloid droplet (c), dilated rough endoplasmic reticulum (rER) and corrugated nucleus with thin rim of heterochromatin (white arrow). The luminal surface demonstrates elongated and detached microvilli (black arrow); $\times 10,000$.

\section{DISCUSSION}

Light at night became an essential part of modern lifestyle. This causes a significant disruption of the daily circadian rhythm [29]. Disruption of circadian rhythm affects many physiological and behavioural functions [13]. Also it is considered a model of stress, because it increases serum concentration of corticosterone [18]. The thyroid gland is very sensitive to stress [20]. All metabolically active cells require $\mathrm{TH}$ for proper functioning. Therefore, thyroid dysfunc- 


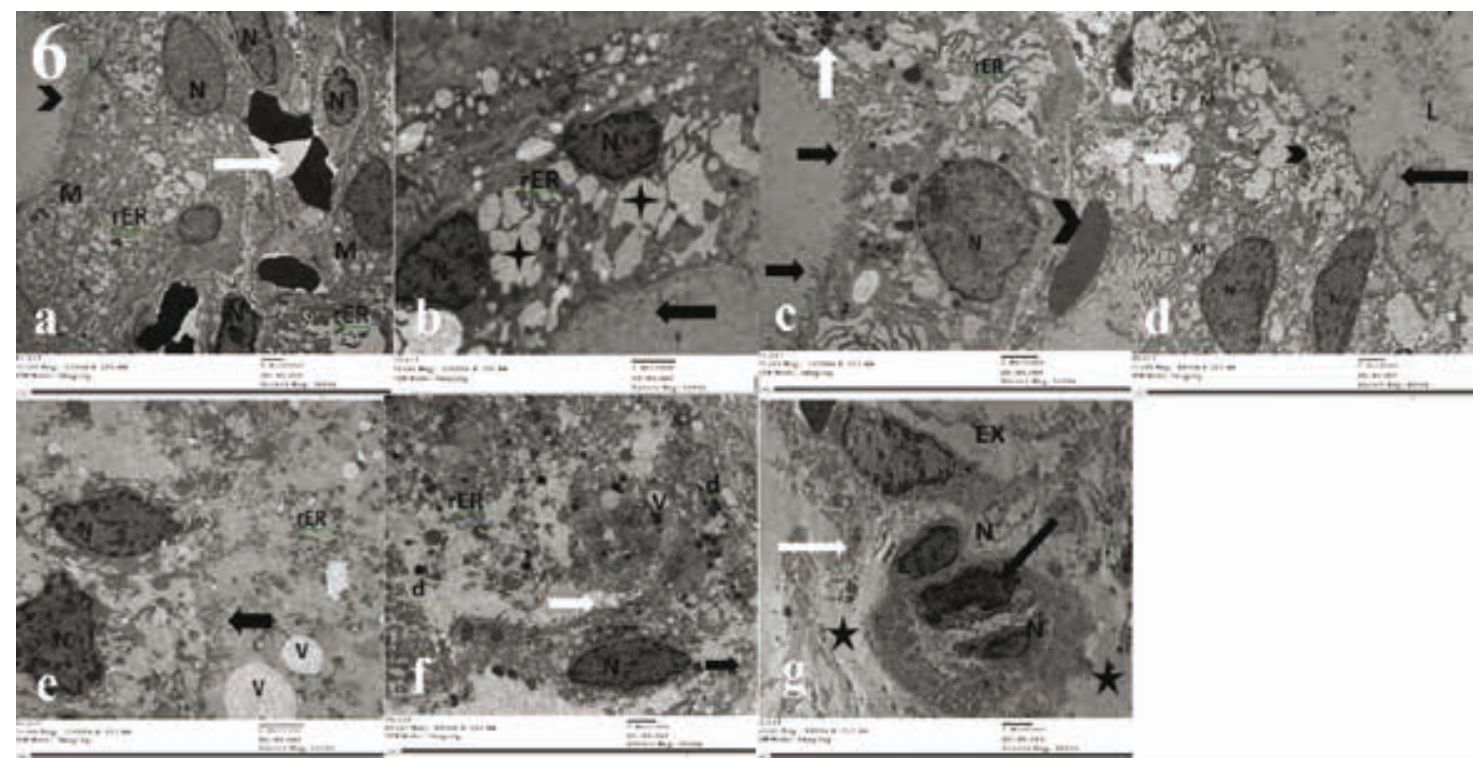

Figure 6. An electron micrograph of the thyroid gland of a prepubertal rat of group III showing: a. Follicular cells with variably shaped hyperchromatic nuclei (N), swollen degenerated mitochondria (M), and fragmented rough endoplasmic reticulum (rER). The luminal surface shows numerous, detached microvilli (arrow head). Note the capillary containing RBCs in the interfollicular stroma (white arrow); $\times 3000 ; \mathbf{b}$. Follicular cells with rarefied cytoplasm (star), fragmented rough endoplasmic reticulum (rER) and hyperchromatic nuclei (N). Note detached apical microvilli in the lumen (black arrow); $\times 6000$; c. Large follicular cell nucleus with dense chromatin at the periphery (N). Electron dense granules similar to lysosomes (white arrow) and fragmented and dilated rough endoplasmic reticulum (rER) are also seen. Note the interfollicular capillary containing RBCs (arrow head) and detached microvilli from the luminal surface (black arrows); $\times 5000$; $\mathbf{d}$. Follicular cells with rarefied cytoplasm (white arrow), swollen degenerated mitochondria (M), fragmented rough endoplasmic reticulum (rER) (arrow head) and hyperchromatic nuclei with marginated condensed chromatin (N). Note the follicular lumen (L) and disrupted apical membrane (black arrow); $\times 4000$; e. Disrupted follicular cells with shrunken, condensed, variably shaped nuclei (N). Cytoplasmic vacuolation (V), fragmented rough endoplasmic reticulum and detached microvilli (black arrow) are exfoliated in the follicular lumen; $\times 6000$; $f$. Ruptured follicular cells with shrunken, hyper chromatic nucleus (N). Note cytoplasmic vacuolation (V), fragmented rough endoplasmic reticulum (rER), electron dense granules (d) similar to lysosomes and detached microvilli are exfoliated in the follicular lumen (white arrow). Thin bundles of collagen fibres (black arrow) are seen; $\times 4000 ; \mathbf{g}$. Exfoliation (EX) of some cells in the fine granular colloid material. The exfoliated cells show condensation of the nuclear chromatin (black arrow) with distorted, variably shaped nuclei (N). Note marked reduction of cell organelles (star) and thin bundles of collagen fibres (white arrow). $\times 4000$.

tion can have a wide range of effects on most of body systems [31]. There is a functional connection between pineal gland, melatonin and the thyroid gland. Melatonin had a strong inhibitory effect on cell proliferation and TH synthesis [25]. This explains the stimulation of thyroid gland under exposure to constant light as it is relieved from the inhibitory effect of melatonin [3].

In the present work, light microscopic findings in rats exposed to constant light for short period (1 month) revealed that the thyroid gland was stimulated, as evidenced by microfollicular thyroid structure and little or no colloid in the thyroid follicles. Similar results were detected by Olatunji and Sofola [30]. Belviranli and Baltaci [3] reported that the activity of the pineal gland increased and thyroid growth was inhibited in male rats under light restriction. This might be explained by increased melatonin secretion in response to dark condition. These results ensured that the thyroid gland is stimulated in constant light exposure (as in the present work) and inhibited in dark condition.

In the current study, rats exposed to constant light for long period (3 months) had overstimulated glands, some of which exhibited hyperplastic changes. The later findings were novel in this work. The follicles exhibited back to back arrangement (signs of hypertrophy with hyperplasia). Occasionally, the follicles were lined by multiple layers of follicular cells. Diffusely hyperplastic thyroid was detected in the form of follicles lined by tall columnar epithelium and the crowded epithelial cells projected into the lumen. These cells were actively resorbing the colloid. This result was comparable with Milosevic et al. [28] who reported that prolonged exposure to light of 600 lux (the same intensity was used in the present study) activated stress system and stimulated growth and hypertrophy of adrenal zona fasciculata cells in female rats. In this regard, Miler et al. [27] mentioned that exposure to $\mathrm{CL}$ diminished nocturnal 
increase in pituitary deiodinase type 2 which converted $\mathrm{T} 4$ to biologically active $\mathrm{T} 3$. The decrease in this enzyme lowered circulating T3 levels. Therefore, we can conclude that the compensatory increase in secretion of TSH caused follicular cell hypertrophy and hyperplasia. Therefore, we can hypothesize that the function of thyroid gland was stimulated in the short duration group (group II). This was consistent with Gooley et al. [15] who reported that thyroid was relieved from the inhibitory effect of melatonin, as exposure to constant light suppressed its secretion. It might affect thyroid directly or indirectly by affecting hypothalamus-pituitary regulation [12].

On the other hand after prolonged exposure to constant light in the long duration group (group III), thyroid tissue became sensitised to stimulation with TSH. In a contradictory study, Kursawe and Paschke [23] demonstrated that after prolonged exposure to constant light conditions thyroid tissue became desensitised to stimulation with TSH, due to reduced activity and binding capacity of the thyroid tissue TSH receptor. This discrepancy might be attributed to the period and intensity of constant light. Regarding the shape of follicular cells in groups II and III, the majority of cells were high cuboidal or columnar and this finding was in concomitance with Qureshi and Mahmood [32]. This might be attributed to the stimulatory effect of TSH on the follicular cells to synthesize and secrete more hormones into the circulation due to the increased demand for the TH in an attempt to compensate for TH decrease. Degeneration and apoptosis of follicular cells were noticed in groups II and III, as evidenced histologically by dark nuclei and ruptured follicles in some parts with desquamation of cells inside the lumen. This was in accordance with Escribano et al. [11] who reported that exposure to constant light increases cellular oxidative stress, decreases the activity of antioxidants and increases apoptosis inside the gland. In this regard, Tunez and Montilla [38] added that melatonin had anti-oxidative stress and anti-apoptotic effects on rats. Similar findings were reported by Talaber et al. [36] who detected degenerated thymic epithelial cells after constant light exposure. The latter suggested another explanation: accelerated thymic epithelial aging caused by elevated glucocorticoids.

In the current study, most of the follicular cells in group III exhibited cytoplasmic vacuolations, which could be attributed to apoptosis. Similar finding was reported by Escribano et al. [11] who attributed this to increased lipoperoxide levels and reduced anti-oxidative stress biomarkers. In agreement, Rifaai and Abd El Baky [35] demonstrated that 1-month period of constant light caused structural changes in the rat thymus. These changes might cause autoimmune response.

In the present work, the electron microscopic results of group II confirmed the results obtained by the light microscope. The follicular cells had hyperchromatic nuclei, fragmented and dilated rough endoplasmic reticulum ( $r E R$ ), swollen mitochondria with disrupted cristae and electron dense secretory granules. Vacuolated or rarefied cytoplasm has been attributed by Rajkovic et al. [33] to the presence of dilated cisternae of rER. These markedly dilated cisternae of $r E R$ in combination with other cellular changes might be an evidence of disruption to protein synthesis.

Swollen mitochondria with disrupted membrane were observed in both group II and group III in the present study, which might be explained by oxidative stress induced by constant light exposure. This may lead to mitochondrial dysfunction, disruption of their inner membrane with increased permeability which allowed the solute to enter the matrix and lead to its swelling, followed by rupture of the outer mitochondrial membrane and release of the pre-apoptotic proteins. El Mahalawy et al. [10] reported the same finding in renal tubular cells. Zhang et al. [43] added that melatonin counteracted oxidative damage of mitochondrial DNA and restored the mitochondrial respiratory control system. So melatonin deficiency due to constant light in this work might be an explanation.

The electron microscopic results of group III showed that some follicles were lined by high cuboidal cells with clumping of peripheral heterochromatin. Their cytoplasm contained several vacuoles, colloid vesicles, and dense lysosomes. Massively dilated cisternae of $r E R$ and loss of their lamellar arrangement were also observed. Their apical borders showed detached aggregated microvilli. In some cells, nuclei appeared irregular in shape, shrunken with condensed heterochromatin. Also, follicular hyperplasia may be connected with a cascade of cellular events involving oxidative stress, genomic DNA damage, and modulation of apoptotic regulatory gene after exposure to constant light [43].

In the current study, exfoliated follicular cells with indistinct organelles in the lumen were observed in group III. These ultrastructural findings confirmed the light microscopic results and indicated that prolonged 
exposure to constant light could induce follicular cell damage and follicular disruption. This result could be attributed to apoptosis and thyroid toxicity explained by Escribano et al. [11]. Congested blood vessels observed in this study were in accordance with that reported by Yu et al. [41] and Hassanin et al. [17]. They explained that oxidative stress and lipid peroxidation might affect vascular walls, leading to their dilatation and congestion.

The present work revealed histological changes in the parafollicular (C cells) cells in group III that were evident at the ultrastructural level. The cells showed swollen mitochondria, few ribosomes, hyperchromatic nucleus and few lysosomes. These observations indicated the possibility of a relationship between the functional state of the thyroid gland and the activity of $C$ cells. It was suggested that changes in $C$ cells were in line with the alterations in the follicular cells. Considering that; the elevated TSH level might directly regulate $C$ cells [26].

\section{CONCLUSIONS}

In the current work, it can be concluded that short-term exposure to constant light for 1 month had minimal effect on thyroid gland tissues (microfollicular structure with decreased luminal colloid and hormonal picture of early hypothyroidism). While longer exposure to light for 3 months had detrimental effects on the thyroid gland structure of male albino rats (hyperplastic and degenerative changes). To the best of our knowledge, this is the first report demonstrating that $\mathrm{CL}$ induced hyperplastic changes in the thyroid gland. These effects were more evident in the adult groups.

\section{REFERENCES}

1. Allan JS, Czeisler CA. Persistence of the circadian thyrotropin rhythm under constant conditions and after light-induced shifts of circadian phase. J Clin Endocrionol Metab. 2010; 79(2): 508-512, doi: 10.1210/jc.79.2.508.

2. Azevedo LH, Aranha AC, Stolf SF, et al. Evaluation of low intensity laser effects on the thyroid gland of male mice. Photomed Laser Surg. 2005; 23(6): 567-570, doi: 10.1089/ pho.2005.23.567, indexed in Pubmed: 16356148.

3. Belviranli M, Baltaci KA. The relation between reduced serum melatonin levels and zinc in rats with induced hypothyroidism. Cell Biochem Funct. 2008; 26: 19-23.

4. Benstaali C, Mailloux A, Bogdan A, et al. Circadian rhythms of body temperature and motor activity in rodents their relationships with the light-dark cycle. Life Sci. 2001; 68(24): 2645-2656, indexed in Pubmed: 11400908.

5. Boelaert K, Franklyn JA. Thyroid hormone in health and disease. J Endocrinol. 2005; 187(1): 1-15
6. Castelhano C, Baumans V. The impact of light, noise, cage cleaning and in-house transport on welfare and stress of laboratory rats. J Sci. 2009; 43: 311-327.

7. Chi HC, Chen SL, Liao CJ. Thyroid hormone receptors promote metastasis of human hepatoma cells via regulation of regulation of trail. J Cell Death Differ. 2012; 19: 1802-1814.

8. Coomans $C P$, van den Berg SAA, Houben T, et al. Detrimental effects of constant light exposure and high-fat diet on circadian energy metabolism and insulin sensitivity. FASEB J. 2013; 27(4): 1721-1732, doi: 10.1096/fj.12-210898, indexed in Pubmed: 23303208.

9. Dauchy RT, Dauchy EM, Tirrell RP, et al. Dark-phase light contamination disrupts circadian rhythms in plasma measures of endocrine physiology and metabolism in rats. Comp Med. 2010; 60(5): 348-356, indexed in Pubmed: 21262119.

10. El-Mahalaway A, Salem M, Mousa A. The effect of potassium dichromate on convoluted tubules of the kidney of adult male albino rats and the possible protective role of ginseng. Egypt J Histol. 2015; 38(2): 157-167, doi: 10.1097/01.ehx.0000464738.41270.06.

11. Escribano BM, Díaz-Moreno A, Moreno A, et al. Impact of light/dark cycle patterns on oxidative stress in an adriamycin-induced nephropathy model in rats. PLoS One. 2014; 9(5): e97713, doi: 10.1371/journal.pone.0097713, indexed in Pubmed: 24852173.

12. García-Marín R, de Miguel M, Fernández-Santos JM, et al. Melatonin-synthesizing enzymes and melatonin receptor in rat thyroid cells. Histol Histopathol. 2012; 27(11): 1429-1438, doi: 10.14670/HH-27.1429, indexed in Pubmed: 23018242.

13. Ghadiri E, Ahmadi R, Nargesi A. Effects of Darkness Stress on Thyroid Function. International Conference on Chemical, Agricultural and Medical Sciences (CAMS-2014) May 2-3, 2014 Antalya (Turkey). 2014, doi: 10.15242/iicbe.c514077.

14. Gibbons R, Hankey J. Influence of vertical illuminance on pedestrian visibility in crosswalks. Trans Res Record. 2009; 1973(1): 105-112, doi: 10.1177/0361198106197300113.

15. Gooley J, Chamberlain K, Smith K, et al. Exposure to room light before bedtime suppresses melatonin onset and shortens melatonin duration in humans. Endocrinology. 2011; 152(2): 742-742, doi: 10.1210/endo.152.2.zee742.

16. Hall LC, Salazar EP, Kane SR, et al. Effects of thyroid hormones on human breast cancer cell proliferation. J Steroid Biochem Mol Biol. 2008; 109(1-2): 57-66, doi: 10.1016/j. jsbmb.2007.12.008, indexed in Pubmed: 18328691.

17. Hassanin KMA, Abd El-Kawi SH, Hashem KS. The prospective protective effect of selenium nanoparticles against chromium-induced oxidative and cellular damage in rat thyroid. Int J Nanomedicine. 2013; 8: 1713-1720, doi: 10.2147/JJN.S42736, indexed in Pubmed: 23658489.

18. Haugen BR. Drugs that suppress TSH or cause central hypothyroidism. Best Pract Res Clin Endocrinol Metab. 2009; 23(6): 793-800, doi: 10.1016/j.beem.2009.08.003, indexed in Pubmed: 19942154.

19. Höfling DB, Chavantes MC, Juliano AG, et al. Low-level laser in the treatment of patients with hypothyroidism induced by chronic autoimmune thyroiditis: a randomized, placebo-controlled clinical trial. Lasers Med Sci. 2013; 28(3): 743-753, doi: 10.1007/s10103-012-1129-9, indexed in Pubmed: 22718472. 
20. Iglesias P, Díez JJ. Thyroid dysfunction and kidney disease. Eur J Endocrinol. 2009; 160(4): 503-515, doi: 10.1530/ EJE-08-0837, indexed in Pubmed: 19095779.

21. Kalsbeek A. Functional connections between the suprachiasmatic nucleus and the thyroid gland as revealed by lesioning and viral tracing techniques in the rat. Endocrinology. 2000; 141(10): 3832-3841, doi: 10.1210/ en.141.10.3832.

22. Kriegsfeld $\sqcup$, Silver $R$. The regulation of neuroendocrine function: Timing is everything. Horm Behav. 2006; 49(5): 557-574, doi: 10.1016/j.yhbeh.2005.12.011, indexed in Pubmed: 16497305.

23. Kursawe R, Paschke R. Modulation of TSHR signaling by posttranslational modifications. Trends Endocrinol Metab. 2007; 18(5): 199-207, doi: 10.1016/j.tem.2007.05.002, indexed in Pubmed: 17524661.

24. Mann DR, Bhat G, Stah CD, et al. Induction of a hypothyroid state during juvenile develop constant light affects pituitary thyroid axis. J Morphol Monkey. 2006; 18: 662-671.

25. Marin GR, Fernandez JM. Melatonin in the thyroid gland: regulation by thyroid-stimulating hormone and role in thyroglobulin gene expression. J Physiol Pharma. 2015; 66(5): 643-652.

26. Martín-Lacave I, Borrero MJ, Utrilla JC, et al. C cells evolve at the same rhythm as follicular cells when thyroidal status changes in rats. J Anat. 2009; 214(3): 301-309, doi: 10.1111/j.1469-7580.2008.01044.x, indexed in Pubmed: 19245497.

27. Miler M, Branka S, Natasa N. Constant light affects pituitary-thyroid axis. J Morph. 2014; 275: 1161-1172.

28. Milosević V, Trifunović S, Sekulić M, et al. Chronic exposure to constant light affects morphology and secretion of adrenal zona fasciculata cells in female rats. Gen Physiol Biophys. 2005; 24(3): 299-309, indexed in Pubmed: 16308425 .

29. Navara KJ, Nelson RJ. The dark side of light at night: physiological, epidemiological, and ecological consequences. J Pineal Res. 2007; 43(3): 215-224, doi: 10.1111/j.1600079X.2007.00473.x, indexed in Pubmed: 17803517.

30. Olatunji BI, Sofola AO. Effect of continuous light and darkness exposures on the pituitary gonadal axis and thyroid activity in male rats. Afr J Biomed Res. 2001; 4: 119-122.

31. Patil, V, Dhurvey, V. Exposure to sodium fluoride affects thyroid follicular cells in albino rats. IJPAES. 2015; 5: 56-61.

32. Qureshi IZ, Mahmood T. Prospective role of ascorbic acid (vitamin C) in attenuating hexavalent chromium-induced functional and cellular damage in rat thyroid. Toxicol Ind Health. 2010; 26(6): 349-359, doi: $10.1177 / 0748233710371109$, indexed in $\mathrm{Pu}-$ bmed: 20504825.
33. Rajkovic V. Light and electron microscopic study of the thyroid gland in rats exposed to power-frequency electromagnetic fields. J Exp Biol. 2006; 209(17): 3322-3328, doi: 10.1242/jeb.02375.

34. Ratcliff R, Van Dongen HPA. Sleep deprivation affects multiple distinct cognitive processes. Psychon Bull Rev. 2009; 16(4): 742-751, doi: 10.3758/PBR.16.4.742, indexed in Pubmed: 19648462.

35. Rifaai AR, Abd El Baky FA. Histological and immunohistochemical study on the effect of constant light exposure on T lymphocyte subsets in the thymus and lymph node of male albino rats. J Histol. 2017; 40(1): 1110-1159.

36. Talaber G, Kvell K, Varecza Z, et al. Wnt-4 Protects Thymic Epithelial Cells Against Dexamethasone-Induced Senescence. Rejuvenation Res. 2011; 14(3): 241-248, doi: 10.1089/rej.2010.1110.

37. Thakkar BP, Zala VM, Ramachandran AV. Simultaneous melatonin administration effectively deprograms the negative influence of neonatal hypothyroidism on immature follicles but not on mature follicles and body and ovarian weights. J Endocrinol Metab. 2011; 1: 220-226, doi: $10.4021 /$ jem54w.

38. Tunez I, Montilla P. Neuroprotective role of melatonin against Alzheimer's disease, Huntington's disease and other cerebral disorders. In: Tu ' nez I; Montilla P, editors. Melatonin present and future. Nova Science Publishers Inc., New York 2007: 315-347.

39. Vinogradova IA, Anisimov VN, Bukalev AV, et al. Circadian disruption induced by light-at-night accelerates aging and promotes tumorigenesis in rats. Aging (Albany NY). 2009; 1(10): 855-865, doi: 10.18632/aging.100092, indexed in Pubmed: 20157558.

40. Weber JB, Mayer L, Cenci RA, et al. Effect of three different protocols of low-level laser therapy on thyroid hormone production after dental implant placement in an experimental rabbit model. Photomed Laser Surg. 2014; 32(11): 612-617, doi: 10.1089/pho.2014.3756, indexed in Pubmed: 25265487.

41. Yu Fu, Wang Z, Ju B, et al. Apoptotic effect of organophosphorus insecticide chlorpyrifos on mouse retina in vivo via oxidative stress and protection of combination of vitamins C and E. Exp Toxicol Pathol. 2008; 59(6): 415-423, doi: 10.1016/j.etp.2007.11.007, indexed in Pubmed: 18222074.

42. Zambrano A, García-Carpizo V, Gallardo ME, et al. The thyroid hormone receptor $\beta$ induces DNA damage and premature senescence. J Cell Biol. 2014; 204(1): 129-146, doi: 10.1083/jcb.201305084, indexed in Pubmed: 24395638

43. Zhang J, Wang X, Vikash V, et al. ROS and ROS-Mediated Cellular Signaling. Oxidative Med Cell Longev. 2016; 2016: 1-18, doi: 10.1155/2016/4350965. 